\title{
A Case of Neurally Mediated Syncope Induced by Laughter Successfully Treated With Combination of Propranolol and Midodrine
}

\author{
Makoto AMAKI, ${ }^{1,2}$ MD, Kei KAMIDE, ${ }^{1}$ MD, Shin TAKIUCHI, ${ }^{3} \mathrm{MD}$, \\ Shinichiro NiIzUMA, ${ }^{1} \mathrm{MD}$, Takeshi Horio, ${ }^{1} \mathrm{MD}$, and Yuhei KAWANO, ${ }^{1} \mathrm{MD}$
}

\begin{abstract}
SUMMARY
A 69-year-old man had been suffering from recurrent syncope induced by laughter since the age of 58 . His syncope was reproduced by head-up tilt testing with isoproterenol infusion and we concluded that his laughter-induced syncope was one type of neurally mediated syndrome (NMS). His daughter also had NMS and her syncope was treated with

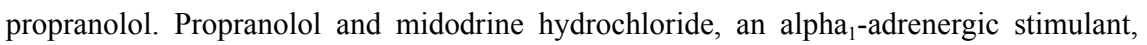
were effective at preventing his laughter-induced syncope. This is a case report of laughter-induced syncope with a familial predisposition successfully treated with the combination of the nonselective beta-blocker propranolol and the alpha ${ }_{1}$-stimulator midodrine.
\end{abstract}

(Int Heart J 2007; 48: 123-127)

Key words: Laughter-induced syncope, Neurally mediated syndrome, Propranolol, Midodrine

SYNCOPE, defined as a transient loss of consciousness, is quite common. In the Framingham study, approximately $3 \%$ subjects had at least one syncope event over a study period of 26 years. ${ }^{1)}$ The most frequent type of syncope is of neurocardiogenic origin (21.2\%) and is known as neurally mediated syncope (NMS). ${ }^{2)}$ Although its prevalence is very common, the mechanisms of NMS are very complex and not fully understood. ${ }^{3)}$ We experienced a case of recurrent syncope after laughter in a patient with a family history of NMS. His syncope was reproduced by head-up tilt testing, and the syncope was successfully treated with a combination of propranolol and midodrine hydrochloride, an alpha ${ }_{1}$-adrenergic stimulant.

\section{CASE REPORT}

A 69-year-old man had been suffering from recurrent syncope since the age

From the ${ }^{1}$ Division of Hypertension and Nephrology, ${ }^{2}$ Division of Cardiology, National Cardiovascular Center, Suita, Osaka, ${ }^{3}$ Division of Cardiology, Higashi-Takarazuka Satoh Hospital, Takarazuka, Hyogo.

Address for correspondence: Kei Kamide, MD, Division of Hypertension and Nephrology, Department of Medicine, National Cardiovascular Center, Suita, Osaka 565-8565, Japan.

Received for publication August 8, 2006.

Revised and accepted December 11, 2006. 
of 58. When he was 61 years old, he was admitted to hospital and diagnosed with NMS by head-up tilt testing with isoproterenol infusion. Treatment with propranolol was started, but discontinued afterwards. A syncope attack occurred once every 2 years, and was always induced by hearty laughter. There were no prodromal symptoms such as coughing before the syncope. After the attack, there were no seizure-like movements, or bowel or bladder incontinence. The subject always regained consciousness after a few seconds. He had no episodes of narcolepsy or sleeping disturbance. He had no other medical history. Regarding his family history, his daughter also had NMS, treated with propranolol for NMS, and second degree atrial-ventricular block, treated with a permanent pacemaker. She was diagnosed with NMS at age 30 by head-up tilt testing with isoproterenol infusion. It was unclear whether or not her syncope could be induced by laughing. Other relatives, including 2 sons and a grandchild, had no obvious history of syncope and so were not examined.

The subject was admitted to our hospital for treatment of his recurrent syncope. He was $156 \mathrm{~cm}$ tall and weighed $74.2 \mathrm{~kg}$. His body mass index was $30.5 \mathrm{~kg} /$ $\mathrm{m}^{2}$, indicating obesity. The results of most of his physical examinations were normal, although a pansystolic Levine II/VI murmur was heard on cardiac auscultation. His blood pressure (BP) at rest was $110 / 72 \mathrm{mmHg}$ and pulse rate (PR) was regular $(56 \mathrm{bpm})$. An electrocardiogram (ECG) was normal, and a 24-hour Holter ECG showed neither arrhythmia nor bradycardia leading to syncope. Mild mitral regurgitation was detected by echocardiography. The results of other examinations, including electroencephalography, cranial computed tomography, and carotid artery ultrasonography, were normal. To rule out ischemic heart disease, dipyridamole-loading cardiac radionucleotide imaging was performed, but the result was negative. Since the syncope occurred during laughing, we assumed he had a hypersensitive carotid sinus, but carotid artery massage did not lead to hypotension or syncope. Since there was no laughter-induced syncope in the hospital and NMS was suspected, a head-up tilt table (HUT) test was conducted (Figure 1A). After resting in a supine position for 20 minutes, the patient was tilted at an angle of 70 degrees for 40 minutes. As neither syncope nor hypotension was induced, the tilt table was returned to the horizontal position and an infusion of isoproterenol was started with an initial dose of $0.01 \mu \mathrm{g} / \mathrm{kg} / \mathrm{min}$. After his heart rate had been stabilized at $75 \mathrm{bpm}$ (supine), representing a $20 \%$ increase in baseline PR, with isoproterenol at $0.02 \mu \mathrm{g} / \mathrm{kg} / \mathrm{min}$, his BP dropped from 123/52 to 86/ $47 \mathrm{mmHg}$. Since no symptoms occurred at this point, the upright tilt at an angle of 70 degrees was repeated. Within a few seconds, overt syncope occurred and the tilt table was immediately returned to a horizontal position. He regained consciousness and his BP returned to normal immediately. At this time, he reported 


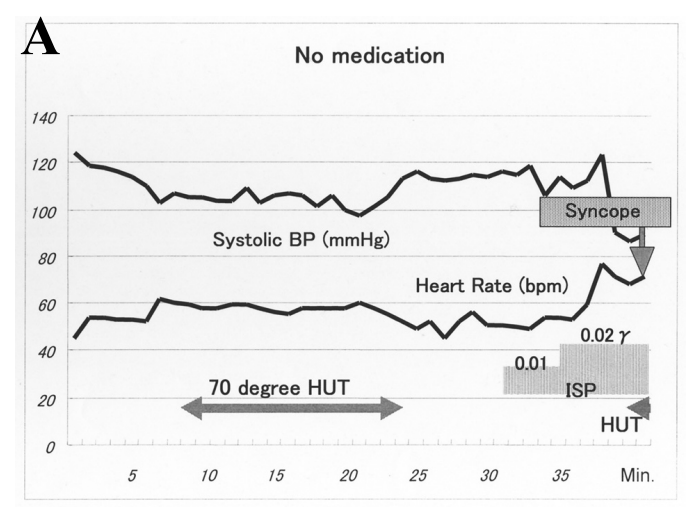

\section{B}

Atenolol $75 \mathrm{mg}$
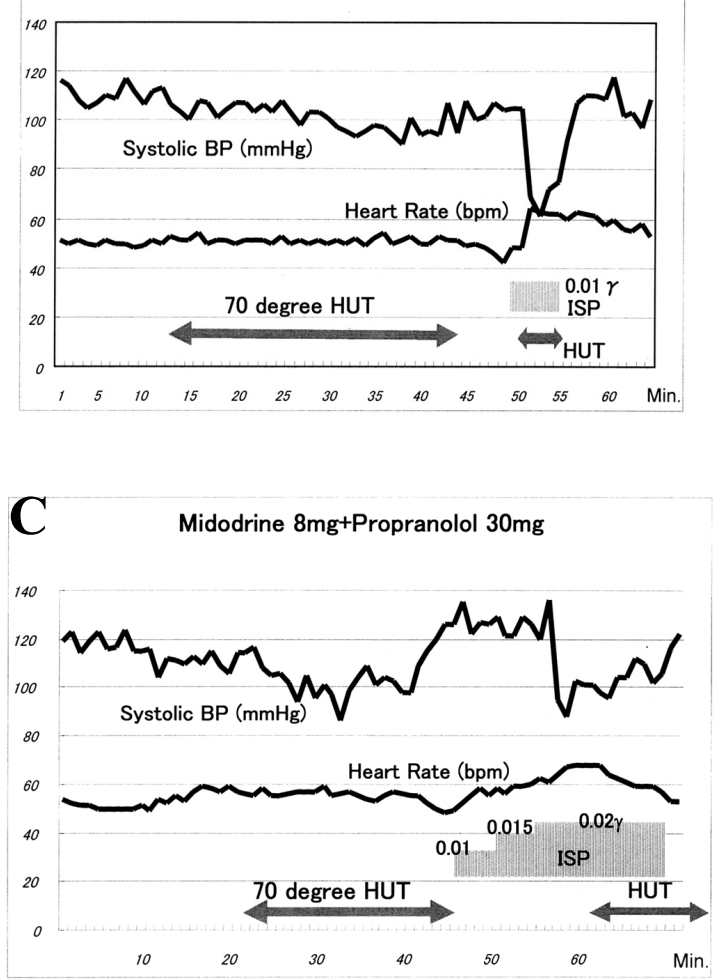

Figure 1. A: Clinical course in the head-up tilt table test when the patient had taken no medication. The syncope occurred after the infusion of $0.02 \gamma(\mu \mathrm{g} / \mathrm{kg} / \mathrm{min})$ of isoproterenol and at an angle of 70 degrees. BP indicates blood pressure; HUT, head-up tilt; and ISP, isoproterenol infusion. B: Clinical course in the head-up tilt table test when the patient was taking atenolol (75 mg/day). Presyncope and hypotension (systolic BP, $60 \mathrm{mmHg}$ ) occurred after the infusion of $0.01 \gamma(\mu \mathrm{g} / \mathrm{kg} / \mathrm{min})$ of isoproterenol and at an angle of 70 degrees. C: Clinical course in the head-up tilt table test when the patient was taking midodorine hydrochloride ( $8 \mathrm{mg} /$ day $)$ and propranolol (30 mg/day). A temporary drop in blood pressure (systolic BP, $60 \mathrm{mmHg}$ ) occurred after the infusion of $0.02 \gamma(\mu \mathrm{g} / \mathrm{kg} / \mathrm{min})$ of isoproterenol and at an angle of 70 degrees, but blood pressure immediately recovered. Syncope was not induced in this test with these medications. 
a feeling similar to his syncope induced by laughter. Based on this result, we diagnosed him with laughter-induced NMS.

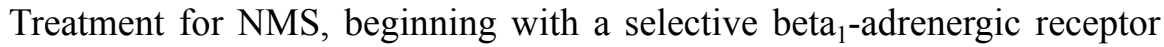
blocker (atenolol $75 \mathrm{mg}$ /day), was started. After a week on this medication, his basal PR averaged $50 \mathrm{bpm}$ and the tilt table test was performed to evaluate the efficacy of the treatment (Figure 1B). When isoproterenol $0.01 \gamma$ was infused with the tilt table at 70 degrees, his systolic BP dropped from 105 to $60 \mathrm{mmHg}$. He felt drowsy but did not lose consciousness and eventually could no longer stand by himself. Given the PR response during the tilt table test and that he had experienced syncope when taking a nonselective beta-adrenergic blocker, propranolol, we speculated that the episodes were partially due to vasodilatation and that propranolol was not strong enough to prevent his syncope. Therefore, we attempted administration with a selective alpha $\mathrm{a}_{1}$-adrenergic receptor agonist, midodrine hydrochloride. The tilt table test was performed again with the patient taking propranolol $30 \mathrm{mg} /$ day and midodrine hydrochloride $8 \mathrm{mg}$ /day administered in combination. His systolic BP dropped to $90 \mathrm{mmHg}$, however, it increased immediately, and syncope was not induced even by the infusion of isoproterenol $0.02 \gamma$ (Figure 1C). He was discharged on midodrine hydrochloride and propranolol, and advised to refrain from intense laughter in the future. In the two years he has been attending our outpatient clinic, he has had no attacks of syncope. His syncope was not induced by the Valsalva maneuver after taking midodrine and propranolol, although the maneuver had not been attempted before taking medication.

\section{Discussion}

There have been a number of reports of syncope related to laughter. Recently, Bloomfield, et al reported that a healthy 32 year-old male experienced laughter-induced syncope after listening to a funny story. ${ }^{4}$ Braga, et al also reported the case of a 63 year-old man with laughter-induced syncope, ${ }^{5)}$ which was reproduced by the Valsalva maneuver. Laughter-induced syncope is probably analogous to tussive syncope or other types of syncope associated with increased intrathoracic or intra-abdominal pressure via a Valsalva mechanism. ${ }^{4)}$ According to blood pressure and heart rate recordings in the case report by Braga, et al, ${ }^{5)}$ lack of activation of the sympathetic nervous system in the late stage of phase II in the Valsalva maneuver might be the main cause of laughter-induced syncope. Laughter-induced syncope is very similar to cataplexy, which is a component of, and highly specific to narcolepsy. ${ }^{6}$ Thus, laughter-induced syncope may be mistaken for cataplexy. In cases of cataplexy, emotion, rather than the physical act of laughing, is thought to trigger an attack. ${ }^{7}$ Since the syncope in our case was 
reproduced by HUT with an infusion of isoproterenol with a similar feeling of syncope, we concluded that his laughter-induced syncope was very similar to NMS.

To the best of our knowledge, no therapeutic approaches have been established for laughter-induced syncope. Our patient appears to represent the first reported case of laughter-induced syncope successfully treated with midodorine hydrochloride, an alpha ${ }_{1}$-stimulator, and propranolol. The alpha ${ }_{1}$-stimulator increases venous return to the heart through the vasoconstriction of veins and elevates blood pressure via the vasoconstriction of peripheral arteries. As a consequence, it could increase brain blood flow and prevent the NMS. The effectiveness of midodrine for NMS has been reported in a double-blind randomized, crossover study. ${ }^{8)}$ We speculate that laughter-induced syncope could be diagnosed as NMS and the combined administration of a nonselective betablocker and alpha ${ }_{1}$-stimulator might be useful in preventing this syncope.

\section{REFERENCES}

1. Soteriades ES, Evans JC, Larson MG, et al. Incidence and prognosis of syncope. N Engl J Med 2002; 347: 87885.

2. Rossen R, Kabat H, Anderson JP. Acute arrest of cerebral circulation in man. Arch Neurol Psychiatr 1943; 50: 510-28.

3. Fenton AM, Hammill SC, Rea RF, Low PA, Shen WK. Vasovagal syncope. Ann Intern Med 2000; 133: 71425. (Review)

4. Bloomfield D, Jazrawi S. Shear hilarity leading to laugh syncope in a healthy man. JAMA 2005; 293: $2863-4$.

5. Sarzi Braga S, Manni R, Pedretti RF. Laughter-induced syncope. Lancet 2005; 366: 426.

6. Totah AR, Benbadis SR. Gelastic syncope mistaken for cataplexy. Sleep Med 2002; 3: 77-8.

7. Aldrich MS. Diagnostic aspects of narcolepsy. Neurology 1998; 50 (2 Suppl 1) : S2-7. (Review)

8. Kaufmann H, Saadia D, Voustianiouk A. Midodrine in neurally mediated syncope: a double-blind, randomized, crossover study. Ann Neurol 2002; 52: 342-5. 